ESAIM: PROCEEDINGS, October 2007, Vol. 22, 198-199

Gabriel Caloz \& Monique Dauge, Editors

\title{
A FOREWORD TO THE MOLECULAR SIMULATION MINI-SYMPOSIUM
}

\author{
Philippe Chartier ${ }^{1}$
}

\begin{abstract}
Molecular simulations have become essential tools in the understanding of materials at the atomistic level with numerous applications in science and engineering. This text is a brief introduction to the two papers presented during the molecular simulation mini-symposium.
\end{abstract}

Molecular simulations have become essential tools in the understanding of materials at the atomistic level with numerous applications in science and engineering (molecular biology, nano-technology, ...). They involve a broad range of techniques and methodologies such as molecular dynamics, Monte-Carlo methods, Brownian dynamics, ... based on more or less sophisticated description of the underlying physical and chemical phenomena

At the finest level, Schrödinger equations (and approximations of it) describe the state and the evolution of molecular systems very accurately. Given that they can not be solved analytically, their solutions require numerical simulations. Hence follows a broad range of models, from the most elaborated ones at quantum scale $a b$ initio to more rudimentary ones such as those based on classical mechanics with force potentials. The first ones consist in solving minimization problems of the form

$$
\inf \{<H \phi, \phi>,<\phi, \phi>=1\}
$$

in order to find the fundamental state of the system, or, alternatively, to simulate the dynamics of the system through the time-dependent Schrödinger equation

$$
i \hbar \frac{\partial \phi}{\partial t}=H \phi
$$

Such systems are incredibly complex and their solution is a formidable task which requires specific algorithms. In practice, they are limited to very small systems for which an accurate description is required.

The second ones ignore the exact position of electrons and consider the atoms as aggregates of kernels and electrons (the presence of electrons is taken into account through an average force field which modifies in an appropriate way the interactions of atoms). In its statistical form, called molecular mechanics, it comes to minimizing

$$
\inf V\left(x_{1}, x_{2}, \cdots, x_{M}\right)
$$

\footnotetext{
1 INRIA, Campus de Beaulieu, 35042 Rennes Cedex, France; e-mail: chartier@irisa.fr
}

(c) EDP Sciences, SMAI 2007 
in order to find the equilibrium configuration of the the kernels. In its dynamical form (called molecular simulation), it consists in simulating Newton's equations

$$
m_{i} \frac{d^{2}}{d t^{2}} x_{i}=\nabla_{x_{i}} V\left(x_{1}, x_{2}, \cdots, x_{M}\right)
$$

At every stage of this cascade of models, one is confronted with mathematical and numerical difficulties. The works presented in this mini-symposium reflect a few of them in some specific situations and for one or the other approach described above.

\section{REFERENCES}

[1] D. Frenkel and B. Smit, Understanding Molecular Simulation, Computational Science Series, Vol 1, AAP, 1982.

[2] P. Balbuena, J. Seminario editors, Molecular Dynamics, From Classical to Quantum Methods, Elsevier 1999.

[3] E. Hairer, C. Lubich, G. Wanner, Geometric Numerical Integration. Structure-preserving Algorithms for Ordinary Differential Equations. Springer, Berlin, 2002.

[4] B. Leimkuhler, S. Reich, Simulating Hamiltonian dynamics. Cambridge Monographs on Applied and Computational Mathematics, 14. Cambridge University Press, Cambridge, 2004.

[5] F. Legoll, M. Luskin and R. Moeckel, Non-ergodicity of the Nose-Hoover Thermostatted Harmonic Oscillator, to appear in Archive for Rational Mechanics and Analysis.

[6] E. Cancès, F. Legoll and G. Stoltz, Theoretical and numerical comparison of some sampling methods for molecular dynamics, to appear in Mathematical Modelling and Numerical Analysis.

[7] G. Ciccotti, T. Lelièvre and E. Vanden-EijndenSampling, Boltzmann-Gibbs distributions restricted on a manifold with diffusions, to appear in Communications on Pure and Applied Mathematics. 